\title{
Collaborative Design Automation Toolkit for Power Controllers in Aerospace Applications
}

\author{
Janis Sebastian Häseker ${ }^{1}$ and Niklas Aksteiner ${ }^{1}$ \\ ${ }^{1}$ Institute of Space Systems, German Aerospace Center (DLR), 28359 Bremen, Germany \\ janis.haeseker@dlr.de
}

\begin{abstract}
In this work we present the latest status on our rapid design process and automated toolkit for aerospace power controllers. The goal is to generate correct-by-design flight hardware from high level requirements with a minimum of manual engineering work. This is achieved by maintaining a database of readily usable design elements (circuit designs, PCB layouts, simulation models and documentation snippets). In this paper we focus on the different roles of human interaction with the toolkit and how we can streamline the process to final flight worthy hardware by parallelizing single design tasks.
\end{abstract}

Keywords: Design Automation, Power Systems, Aerospace.

\section{Introduction}

The aerospace sector is known for its stringent requirements towards the reliability of avionic systems. Oftentimes this leads to the reuse of existing designs and heritage from previously deployed systems. This contradicts the path of the space industry to highly innovative, smaller and cheaper missions and ambitious project timelines. For these scenarios a design process is needed to provide bespoke avionics under mass and budget limits without sacrificing reliability.

This can only be achieved if the manual labor is supported by an automated design process. However not all manual engineering tasks can be automated, so it is advantageous to establish a process where this work can be done in a parallelized fashion.

All these factors are addressed by our design process and automation toolkit for aerospace power controllers. It supports the path from high level requirements of the power system, architecture definition, detailed design of circuits, the verification and testing, as well as the manufacturing and integration. This approach follows the philosophy of Platform Based Design, where the design process is understood as a series of refinement steps from the highest level of requirements to the final product using elements designed in bottom-up approach[1]. 


\section{State of the Art}

Today the design of avionic systems for aerospace applications is most of the time a labor intensive process. This is due to the massive amount of documentation combined with the use of a lot of different engineering tools that are not directly interconnected. In the case of spacecraft power systems the design starts with a set of requirements provided by the system engineering team. A preliminary architecture is defined that often depends heavily on the reuse of previously designed units. If the requirements cannot be fulfilled with existing designs single changes to the previous version are proposed. There is a strong focus to keep the design as close as possible to previously flown solutions to ensure that the new unit can most likely be reliable as the old one by means of similarity. This is called heritage in the space industry and oftentimes has a big impact on decisions which component to use, even if there are choices with higher performance or lower price. This slows down innovation massively. If innovative and new designs are used, extensive qualification campaigns are necessary. These are highly expensive and time consuming.

Additionally detailed information on the single avionic products can be included in system studies only late in the project, if these products are developed in parallel. If this is true for multiple products the system design cannot be optimal and the risk of late changes with high impact on cost and schedule arises.

We want to tackle these shortcomings in the traditional design process by redefining how heritage is handled in the design phase. The introduction of design automation techniques with an integrated design tool offers potential for a reliable and fast design of power controllers with a high level of maturity right from the start. In the following sections we introduce our collaborative design automation toolkit, its role in the design of aerospace power controllers and how it supports the collaboration of different engineering disciplines.

\section{$3 \quad$ Proposed Design Process and Toolkit}

\subsection{Overview}

The classical way to start the design of a new avionic product is to check if existing designs can satisfy the requirements. This step can be automated if the requirements and performance indicators are captured in a way that can be processed by machines. We split up the overall system into blocks that represent recurrent functions for power controller design. In this way we can not only check if an old design of the overall power controller can be used but also if a new combination of previously designed functions can fulfill the requirements. These building blocks are kept in a database that acts as the central part of our process. It contains pre-designed implementations of power system functions such as DC-DC converters or load switches. Each entry consists of a set of important performance parameters, simulation models with varying levels of fidelity, printed circuit board layouts and other production information, as well as snippets for the auto-generation of documentation and test plans. 
To lower the barrier of using our system and ensure the most consistent user experience it was decided to build a web application. In this way we can ensure that no special requirements are enforced on the users working environment.

Our design automation toolkit consists of two components: A web based software solution called the design automation application and a rapid hardware prototyping solution called the PCDU modular breadboard. The software includes the necessary functions to perform subsystem level design task, contribute to system level design and interface to other domain specific tools. The PCDU modular breadboard allows a quick evaluation of PCDU designs in real hardware that can be compared to analysis and simulation results. This direct link between design, prototyping and validation tools ensures that solutions with a high quality can be reached in less time and with fewer resources compared to a classical design process.

\subsection{Interaction of Engineering Roles with the Toolkit}

In the following sections we will illustrate how the different engineering roles interact with this design information and how efficient collaboration is promoted.

\section{Power System Engineer}

The Power System Engineer has the role to ensure that the overall power system with means for power generation, storage and distribution satisfy the system requirements. From this the requirements of the power distribution unit are derived, especially the description of interfaces and their expected behavior. This is shown in Fig. 1.

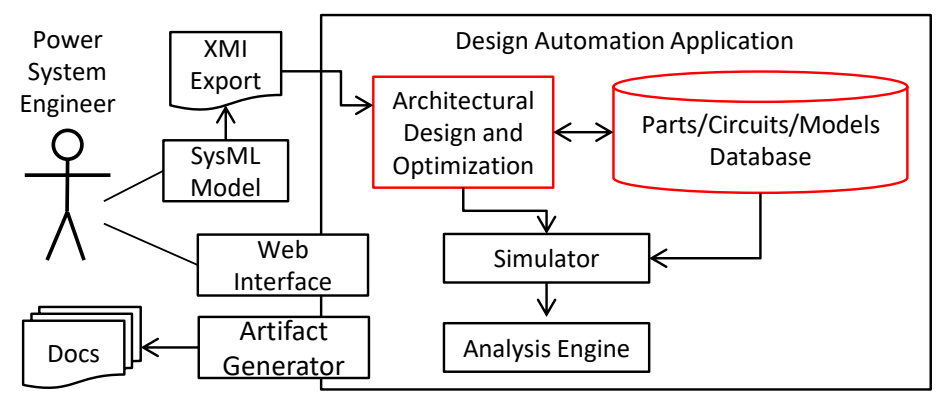

Fig. 1. Interaction of Power System Engineer with the Design Automation Application

The information can be provided via the web interface, XMI exports from SysML system models or domain specific modeling solutions like Virtual Satellite [2]. With a set of requirements and the contents of the database the "Architectural Design and Optimization" (ADO) algorithm can choose and connect a subset of building blocks to provide a possible implementation for the needed power controller functionality. For all generated baseline solutions the system will perform simulations using SystemC-AMS models, and present the analysis results via auto-generated documentation. 
By automating the step of design exploration a much broader design space can be studied and trade-offs between the solutions can be made. Additionally the Power System Engineer gains deeper insight into the design parameters such as mass, volume and electrical efficiency.

\section{Electronic Design Engineer}

The Electronic Design Engineer has the role to maintain the database of circuits that can be used for the design as illustrated in Fig. 2. These entries can have different maturity levels: from a simple simulation model based on datasheet values over more sophisticated circuit simulations up to measurements of real hardware with full documentation and design files. In our application simulations are performed using SystemC-AMS. It allows the application of a wide range of abstraction levels from behavioral description of elements down to circuit level models.

If the ADO process results in non-optimal results in terms of efficiency, mass or volume it is beneficial to manually design new elements for the database. For this Spice models of the circuits are developed that are automatically approximated by SystemC-AMS simulation models used in the application. The engineer also needs to provide documentation templates, to allow the application to produce artifacts like test plans for the newly added element.

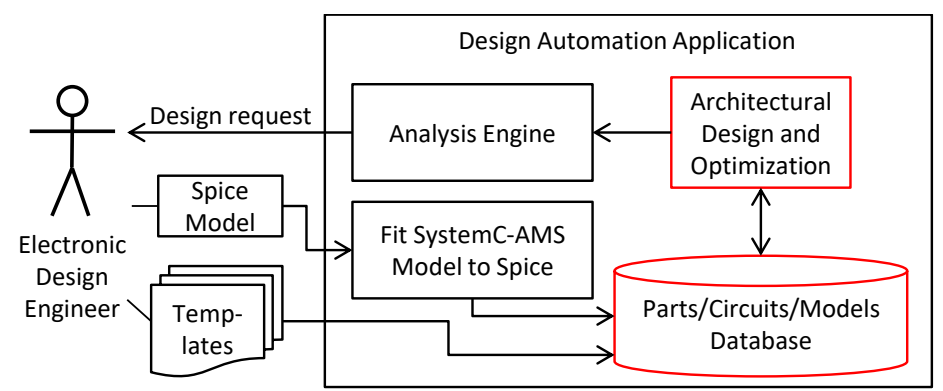

Fig. 2. Interaction of Electronics Design Engineer with the Design Automation Application

\section{Test Engineer}

The role of the Test Engineer is to perform all verification steps on the designed hardware as shown in Fig. 3. For every element in the database there exists an evaluation board that can be used to rapidly assemble a whole power unit using our rapid prototyping solution: the PCDU modular breadboard [3]. It interconnects all the single circuit boards and provides control and telemetry interfaces to provide a fully functional PCDU. The configuration and test plans for this are auto-generated. Measurements from the setup are directly transferred to the database via the Modular Breadboard interface. Additional information of the test performance can be provided via the web interface. This setup not only allows to test the hardware design itself, but also to validate the test procedures that can be later used for the flight models. 


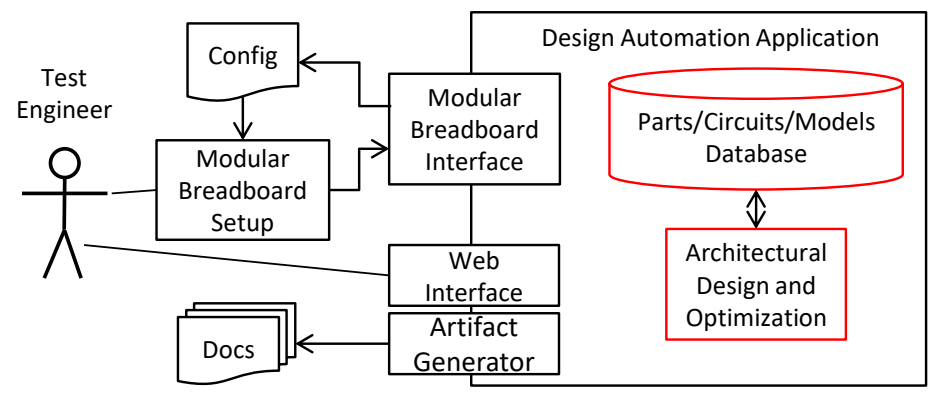

Fig. 3. Interaction of the Test Engineer with the Design Automation Application

\section{Production/Integration Engineer}

In the final step the output is generated to manufacture, verify and use the power controller as illustrated in Fig. 4. This includes printed circuit board layout data, as well as CAD drawings for the enclosure. Also the documentation such as user manual, interface control documents and component lists are automatically generated from documentation snippets that are stored with the used building blocks in the database. In the aerospace sector a big part of the work is related to documentation that can be massively reduced by design automation tools.

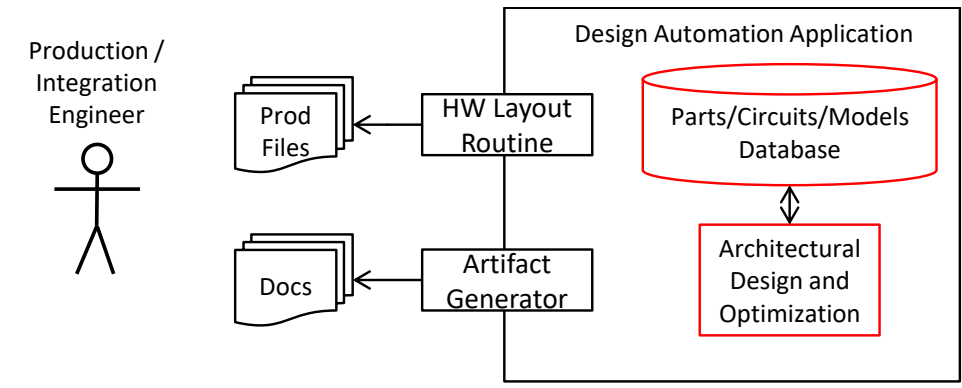

Fig. 4. Interaction of the Production and Integration Engineer with the Design Automation Application

\subsection{Impact on the Engineering Roles}

The overall working scheme promotes a single source of truth on subsystem (PCDU) level inside our web application that is connected to a system level SysML model. This ensures that it is clear where the data for each engineering task can be obtained. To ease the acceptance for our approach we have a strong focus on keeping the work processes that are present in the engineering roles before as similar as possible. The biggest impact can be recognized for the power system engineer. All input to the design tool can be provided via the SysML system model using appropriate attributes, but this model is often not available early in the project. It makes sense to replace previously used preliminary design tools in the form of Microsoft ${ }^{\circledR}$ Excel $^{\circledR}$ sheets or 
Matlab ${ }^{\circledR}$ scripts[4] with the functionality provided by our web tool. In this way much more detailed information can be gathered in terms of first estimates for mass, volume and power losses for the PCDU while satisfying the power system requirements. This ensures a more optimal system design early on. For the electronic design engineer there is no change in the tools, but in the way to share the results of circuit design. Circuit simulations of DC-DC converters and load switches are performed in SPICE with predefined templates for the input and output circuit to ensure compatibility with the simulation engine of the web application. Lookup tables of the simulation results are uploaded to the database together with the original simulation model, performance parameters and documentation snippets. For testing of the different PCDU models the input is mainly auto-generated. This reduces the work load of the test engineer and ensures consistent execution of the verification procedures. Instead of providing written report, test documentation shall be performed inside the web tool. By having a direct connection to the test setup most of the data is gathered automatically. In the end the PCDU must be manufactured using existing supply chains. Here no special tools shall be enforced, especially in the case of external suppliers. We are in the process to collect all the essential data to also auto-generate the necessary design files and documentation which reduces the amount of manual labor to start the production of the final product.

\subsection{Collaborating on Design Tasks}

During the PCDU development there is a strong interaction between the roles of the power system, the electronics design und the test engineer. To keep the process of PCDU design well documented we push towards the sole exchange of information via our web application. In the following we will outline the how collaboration on the design will occur over the different project phases.

During preliminary design of the spacecraft power system the power system engineer needs to quickly iterate on different PCDU designs taking into account the changing system level requirements. Automatically generated PCDU architectures that interconnect functional blocks from the database are analyzed to assess their performance in terms of mass, volume and electrical efficiency. The system tracks if the different building blocks are used and how they perform in the current design. The database contains not only circuits that have been evaluated in hardware, but also mockup elements generated from datasheet values. If these elements are picked during the design process the system generates reports to indicate the need for further investigation towards the electronic design engineer. In this way it can be assured that the focus for the expansion of the catalog of functional blocks will be laid on the ones with the biggest impact for the different projects. This mechanism can be compared to issue tracking systems in software engineering where the most severe bugs are resolved before the work is started on minor problems. By closely tracking the parts chosen for different design trends for certain parameters, combinations can be identified and used for the selection of circuits to be investigated and qualified for use in space in the future. 
When new circuits are designed, evaluation boards with standardized interfaces are manufactured. These can be used for functional and environmental (e.g. radiation, EMC) testing but also to assemble a fully functional model of the PCDU using our PCDU Modular Breadboard. We foresee to directly generate test plans on single circuit and PCDU unit level. With our application being the place where simulation and measurement results with the associated models and test plans are stored, a continuous automated check between simulation and hardware can be performed. This allows all parties to quickly react to deviations between high and low level design as well as measurements on hardware. We foresee that the application can directly connect to the hardware setups allowing test plans to be automatically executed and resulting measurements data to be acquired to ensure repeatability.

Because most verification and test strategies are developed using one tool it eases the communication and collaboration on these topics between the system side and the people that conduct the test activities. The documentation for external suppliers and the integration and verification team gets generated from the same dataset ensuring consistency. It still needs to be assessed how feedback of generated artifacts is handled most efficiently such that the information loop between all parties can be closed.

\section{Conclusion and Future Work}

In this work we presented our web based design tool for the partly automated design of aerospace power controllers. We illustrated how the different engineering roles interact with the application and how efficient collaboration during the design phases is promoted. The application as well as the PCDU development logic behind it is still under development, but there is first evidence showing that a lot of resources can be saved by applying it to our small satellite projects. By reducing the recurring work for the different engineering roles and try to blend in with established modes of work we ensured high acceptance of our tools.

At the moment the application covers the functionality needed for power system and electronic design engineering. In the upcoming time we will expand the application to cover the full development cycle as outlined in this paper. Especially the interface to our PCDU Modular Breadboard rapid prototyping setup will massively accelerate our design activities. We will deploy the system to be used in upcoming small satellite projects at DLR and use it for the development of PCDUs of different sizes and capabilities.

\section{References}

1. Sangiovanni-Vincentelli, A.: Quo Vadis, SLD? Reasoning About the Trends and Challenges of System Level Design, Proceedings of the IEEE 95-3, pp. 467-506 (2007).

2. Fischer, P. et al, Spacecraft Interface Management in Concurrent Engineering Sessions. In: Cooperative Design, Visualization, and Engineering (CDVE), 11792, pp. 54-63, Springer, Mallorca, Spain (2019). 
3. Häseker, J., Strowik, C., Aksteiner, N., Dannemann, F.: Design Process and Modular Breadboard for Rapid Development of Mission Specific Power Conditioning and Distribution Units, Poster SSC19-P1-16, Small Satellite Conference, Utah State University, Logan, UT, USA (2019).

4. Pedersen, J.: Power System for the Eu:CROPIS Satellite - Results from Design TradeOffs, Analysis, Simulation and Testing, E3S Web of Conferences. 16. 13010. 10.1051/e3sconf/20171613010, 2017. 